\title{
Quadratic Constrained Quadratic Estimation for Distribution Network with Limited Measurements
}

\author{
Cheng Long ${ }^{1}$, Hua Zhang ${ }^{1}$, Lilan Dong ${ }^{2 *}$, Yadi Zhang ${ }^{2}$ and Ruipeng Guo ${ }^{2}$ \\ ${ }^{1}$ Electric Power Research Institute of State Grid Sichuan Electric Power Co., Ltd., Chengdu, Sichuan, 610095, China \\ ${ }^{2}$ College of Electrical Engineering, Zhejiang University, Hangzhou, Zhejiang, 310058, China
}

\begin{abstract}
Practical and efficient state estimation is essential for the safe and stable operation of future smart grid. In rectangular coordinates, distribution system state estimation (DSSE) problem can be described as a sparse quadratic constraint quadratic estimation (QCQE) model, which enhances efficiency of the program implementation for DSSE. However, the scarcity of measurements in distribution network brings a formidable challenge to provide reasonably meaningful estimation of the distribution system state. This paper is concerned about application of QCQE in under-determined distribution network. Observability analysis technique is proposed by considering the characteristics of actual distribution network. The observable islands are recognized accordingly, and then unobservable areas can be accurately defined. Pseudo-measurements are adopted to meet the observability requirement. Simulation on IEEE 33-bus shows effectiveness of the proposed method.
\end{abstract}

\section{Introduction}

By utilizing the redundant real-time measurements provided by supervisory control and data acquisition (SCADA), state estimator provides good estimation of bus voltage phasors. With the development of active distribution network, the integration of distributed generators, electric vehicles and controllable loads brings new opportunities and challenges to the distribution network [1-3]. Therefore, sufficiently accurate and instant state estimation is needed to provide real-time information of distribution network to help operators make correct decisions.

Unlike transmission systems that enjoy a high level of measurement data redundancy, distribution systems have limited measurements that large-scale distribution systems are mostly with poor observability. Researches on DSSE appeared in the 1990s. Much work so far has concentrated on dealing with the unobservable problem that caused by scarcity of real-time measurements. By studying the probability distribution density function of loads and the spatial/temporal correlation between adjacent buses, pseudo-measurements can be generated precisely [4-6]. The non-linear auto-regressive exogenous model is used to describe the load change and to obtain the pseudo-measurement values [7]. Machine learning algorithms forecast the loads which are used as pseudomeasurements in $[8,9]$.

In rectangular coordinates, by introducing intermediate variables and equality constraints, the DSSE problem is modelled as a sparse quadratic constrained quadratic estimation (QCQE) model [10]. This paper adopts the QCQE model, which is convenient for programming implementation, to solve the DSSE problem.

In this paper, the observability analysis method is taken into consideration and a further discussion on application of QCQE model in distribution network is made. Rest of the paper is structured as follows: The second section puts forward a novel observability analysis method which is suitable for distribution networks. Unobservable areas are accurately defined on the basis of the proposed criteria. Then a pseudo-measurement generating model is presented. The third section shows the simulation results on the IEEE 33-bus test system. The forth section summarizes the proposed method.

\section{Distribution network observability and pseudo measurement modelling}

Observability analysis identifies whether a set of available measurements is sufficient to estimate the system state. Network observability analysis is mainly accomplished by numerical [11] and topological [12] approaches. Mathematically network observability is related to the rank of the Jacobian matrix of estimation equations, which is very sensitive to the numerical values of its elements [13]. Topological approaches are widely used because they can make judgment on the type and location of real time measurements without any numerical calculations.

\subsection{Distribution network observability analysis}

The observability analysis method proposed in this paper is based on topological analysis. It seeks to find a maximal

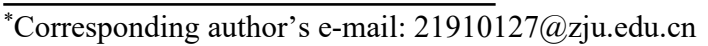


forest of network full rank. When the maximal forest found in the network is a spanning tree, the network is observable. For a distribution network, the feature of radial structure determines that the spanning tree can only be the network itself, which can greatly simplify the existing topological observable methods. Besides, the extensive use of pseudo-measurements is another significant feature of unobservable distribution network. It is important to find the minimal set of pseudomeasurements because excess pseudo-measurements with low precision will degrade the estimation accuracy. Hence, observability analysis needs to accurately detect the unobservable area and efficiently generate pseudomeasurements.

Distribution network is generally running in radial structure. By removing all the branches equipped with branch power flow measurements, the distribution network is divided into several observation areas. The buses connected by remaining branches are naturally assigned into the same observation area. A typical observation area is shown in figure 1.

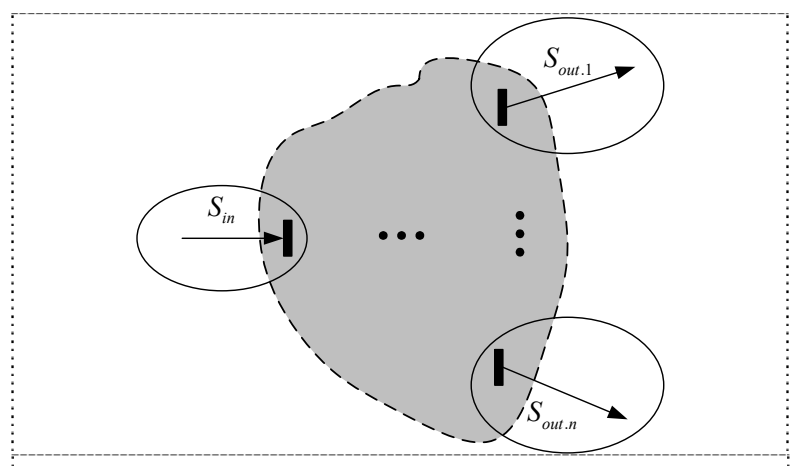

Figure 1. Typical observation area.

Since the power flow of all the branches incident to the observation area are measured, the sum of the total bus injections and loss can be determined:

$$
S_{\text {area }}=S_{\text {in }}-\sum_{i=1}^{n} S_{\text {out } . i}=\sum_{k=1}^{N_{m}} S_{k}+\sum_{l=1}^{N_{u m}} S_{l}+S_{\text {loss }}(\boldsymbol{e}, \boldsymbol{f})
$$

where $S_{\text {in }}$ represents the inflow of upstream branch. $S_{\text {out. } i}$ represents the outflow of downstream branch $i . n$ is the count of the downstream branches. $S_{k}$ and $S_{l}$ denote the injection value of measured bus $k$ and unmeasured bus $l$ respectively. $N_{m}$ and $N_{u m}$ are the number of measured and unmeasured bus injections respectively. $\boldsymbol{e}$ and $\boldsymbol{f}$ represent the real and imaginary part of the vector of bus voltages respectively. $S_{\text {loss }}(\boldsymbol{e}, \boldsymbol{f})$ represents the total power loss of the observation area.

Based on the bus injection measurements, we can take the following criteria for observability analysis:

- If $N_{u m} \leq 1$, namely there is at most one unmeasured bus injection, then the area satisfies the observability requirement. In particular, when all the buses' power injections are measured, the observation area has redundant measurements.
- $\quad$ If $N_{u m} \geq 2$, there are two or more unmeasured bus injections in the observation area which means that the related states are under-determined. Though the total power flow is available, increasing the injection values of some buses can be balanced by reducing that of the others. Therefore, we need to generate pseudo-measurements to avoid multiple solutions.

According to the discussion above, unobservable areas can be detected and then the set of pseudo-measurements are used to recover network's observability.

\subsection{Pseudo measurements modelling}

As a compromise between efficiency and accuracy when augmenting the real-time data with pseudo-measurements:

- By dealing with existing information effectively, pseudo-measurements generated preferably have a good approximate linear relationship. The minimal number of pseudo-measurements can reduce the calculation scale of DSSE, improve the convergence of the algorithm and maintain the QCQE model's computational efficiency.

- The estimated bus injections should be as close as possible to the actual situation, so as to ensure that the accuracy of the state estimation meets the application requirements.

Based on the above discussion, for any unobservable subarea $k$, the total active power, $P_{\Sigma}^{k}$, and reactive power, $Q_{\Sigma}^{k}$, are introduced as new state variables. The pseudomeasurements are calculated as follows:

$$
\left\{\begin{array}{l}
\tilde{P}_{i n j}^{i}=\alpha_{i} P_{\Sigma}^{k} \\
\tilde{Q}_{i n j}^{i}=\beta_{i} Q_{\Sigma}^{k} \quad \forall i \in S_{L}^{k}
\end{array}\right.
$$

where $i$ denotes the number of bus. $\tilde{P}_{i n j}^{i}$ and $\tilde{Q}_{i n j}^{i}$ are the pseudo active and reactive injection measurements of bus $i$. $S_{L}^{k}$ is the collection of all unmeasured bus injections in subarea $k . \alpha_{i}$ and $\beta_{i}$ represent the proportion of the active and reactive power injection of bus $i$ to the total power injection of the observation area.

The proportions are unknown to DSSE, but can generally be approximated by the ratio of injection capacity, and then used in DSSE. In case that energy measurements of a certain duration is known, the proportions approximated by the ratio of active and reactive energy can give better DSSE result.

By identifying the unobservable areas and adding reasonable pseudo-measurements, this section enables distribution networks to meet the observability requirement of DSSE.

\section{Simulation results}

The proposed QCQE model is tested on the IEEE 33-bus test system, shown in figure 2 . The base voltage is 12.66 $\mathrm{KV}$ and the total load is $3715 K W+j 2300 K V A R$. The specific load and parameter information can be obtained from [14]. The load flow results are used to simulate the 
measurement values. No measurement error is introduced in the simulation.

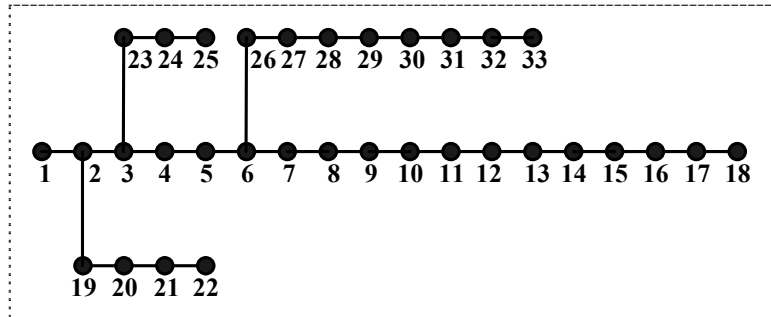

Figure 2. The IEEE 33-bus test system.

In actual distribution network, only the substation and part of the distribution network are equipped with SCADA measurements. Thus, pseudo measurements are provided for state estimation with the proposed pseudo measurement model. The proportions of active and reactive injection powers to related observation area are approximated by the ratio of injection capacity.

The measurement system is configured according to table 1 .

Table 1. The configuration of SCADA measurements.

\begin{tabular}{cc}
\hline Type of measurements & Branch / Bus \\
\hline Bus voltage magnitude $\left(\tilde{U}_{m}\right)$ & $1,2,3,6,12$ \\
Bus power & $23,24,30$ \\
injection $\left(\tilde{P}_{i}, \tilde{Q}_{i}\right)$ & $1-2,2-19,3-23,6-26,11-$ \\
$\operatorname{Branch}$ power & 12 \\
flow $\left(\tilde{P}_{i j}, \tilde{Q}_{i j}\right)$ & \\
\hline
\end{tabular}

According to the rules described in section 2, by removing the branches equipped with power flow measurements, the distribution system is then divided into 6 observation areas as shown in Figure 3. Area A1 contains only one bus, named as bus 1, whose power injection are not measured but can be determined by the measured branch power flows on branch 1-2. Then area A1 is observable. Area A2 contains 3 nodes, but only the power injection of node 25 are not measured, and is also observable. Each of area A3 A6 has more than one bus without injection measurements, and is unobservable. The area power injection states and pseudo-measurements as (2) need be added for DSSE.

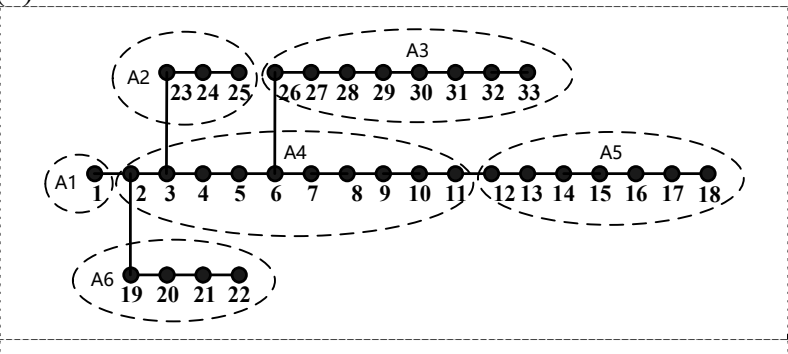

Figure 3. The observation areas of IEEE 33-bus test system.

Figure 4 and figure 5 show the simulated estimation results for the active and reactive power injections on load buses. As to the power injections in observable areas, such as bus 23, 24 and 25 in observation area A2, the estimated values are all quite close to the corresponding true values. As to the bus injections in unobservable areas, the estimated error may much bigger than that of injections in observable areas because of the lower precision of pseudo measurements. As you can see from figure 4 and figure 5 , the estimated values for power injections in unobservable areas are also close enough to the corresponding true values, which are generally precise enough for DSSE.

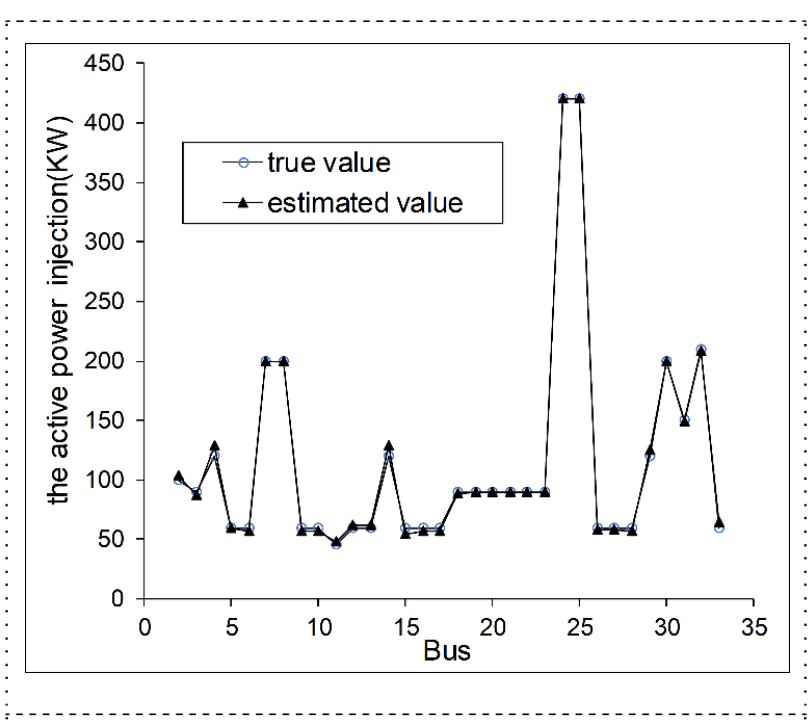

Figure 4. The active power injections on load buses.

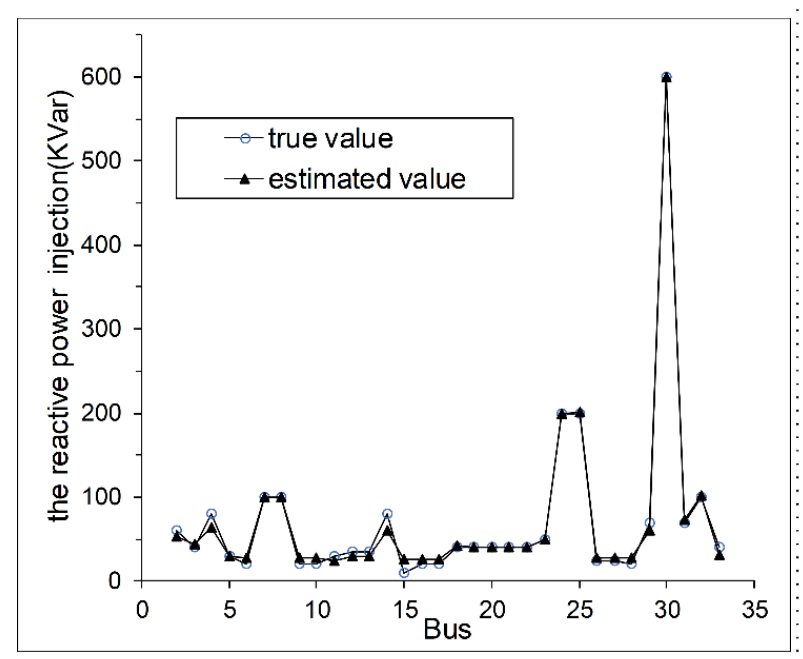

Figure 5. The reactive power injections on load buses.

\section{Conclusions}

This paper proposes an observability analysis method and a related pseudo measurement modelling method for DSSE with limit measurements. The distribution network is divided into observation areas with the branches, which are equipped with power flow measurements, as the boundary. Rules are then proposed to identify the observability of observation areas. Pseudo measurements are introduced for each unobservable area. The QCQE 
model is adopted to solve DSSE problems. Simulation on the IEEE 33-bus test system shows that the proposed observability analysis method is convenient, and the precision of pseudo measurement model is generally acceptable for DSSE.

\section{Acknowledgments}

This paper is supported by the science and technology project of State Grid Sichuan Electric Power Co., Ltd. (521997190014).

\section{References}

1. Primadianto, A., Lu, C. (2017) A Review on Distribution System State Estimation. IEEE Transactions on Power Systems, 32:3875-3883.

2. Ablaković, D., Džafić, I., Jabr, R.A., Pal, B.C. (2014) Experience in distribution state estimation preparation and operation in complex radial distribution networks. In:2014 IEEE PES General Meeting Conference \& Exposition. National Harbor. pp. 1-5.

3. Džafić, I., Jabr, R.A. (2017) Real Time Multiphase State Estimation in Weakly Meshed Distribution Networks with Distributed Generation. IEEE Transactions on Power Systems, 32:4560-4569.

4. Singh, R., Pal, B.C., Jabr, R.A. (2010) Distribution system state estimation through Gaussian mixture model of the load as pseudo-measurement. IET Generation, Transmission \& Distribution, 4: 50-59.

5. Angioni, A., Schlösser, T., Ponci, F., Monti, A. (2016) Impact of Pseudo-Measurements from New Power Profiles on State Estimation in Low-Voltage Grids. IEEE Transactions on Instrumentation and Measurement, 65: 70-77.

6. Nguyen, D.T. (2015) Modeling Load Uncertainty in Distribution Network Monitoring. IEEE Transactions on Power Systems, 30:2321-2328.

7. Hayes, B.P., Gruber, J.K., Prodanovic, M. (2015) A Closed-Loop State Estimation Tool for MV Network Monitoring and Operation. IEEE Transactions on Smart Grid, 6:2116-2125.

8. Manitsas, E., Singh, R., Pal, B.C., Strbac, G. (2012) Distribution System State Estimation Using an Artificial Neural Network Approach for Pseudo Measurement Modelling. IEEE Transactions on Power Systems, 27:1888-1896.

9. Wu, J., He, Y., Jenkins, N., (2013) A robust state estimator for medium voltage distribution networks. IEEE Transactions on Power Systems, 28:1008-1016.

10. Liu, J., Zhu, P., Guo, R. (2018) A Quadratic Constraint Quadratic Estimation Model for ThreePhase Distribution Network State Estimation. In: 2018 2nd IEEE Conference on Energy Internet and Energy System Integration (EI2). Beijing. pp. 1-6.
11. Herrero, H., Solares, C. (2020) A Greedy Algorithm for Observability Analysis. IEEE Transactions on Power Systems, 35:1638-1641.

12. Zhang, H., Han, K. (2018) A Topological algorithm for observability analysis with PMU measurements. In: 2018 International Conference on Power System Technology (POWERCON). Guangzhou. pp. 4431-4437.

13. Zhang, H., Han, K. (2020) A Hybrid Observability Analysis Method for Power System State Estimation. IEEE Access, 8:73388-73397.

14. Goswami, S.K., Basu, S.K. (1992) A new algorithm for the reconfiguration of distribution feeders for loss minimization. IEEE Transactions on Power Delivery, 7: 1484-1491. 